\title{
Os povos indígenas e a Covid-19 nos Distritos Sanitários Especiais Indígenas (DSEI) de Mato Grosso: Um estudo ecológico
}

\author{
Indigenous peoples and Covid-19 in the Special Indigenous Sanitary Districts (DSEI) of Mato \\ Grosso: An ecological study \\ Pueblos indígenas y Covid-19 en los Distritos Sanitarios Especiales Indígenas (DSEI) de Mato \\ Grosso: Un estudio ecológico
}

Recebido: 14/12/2020 | Revisado: 17/12/2020 | Aceito: 20/01/2021 | Publicado: 24/01/2021

Julio Martinez Alves Oliveira

ORCID: https://orcid.org/0000-0002-3173-9444 Universidade Estadual Paulista "Júlio de Mesquita Filho", Brasil E-mail: juliooliveira1994@hotmail.com

Suzely Adas Saliba Moimaz

ORCID: https://orcid.org/0000-0002-4949-529X Universidade Estadual Paulista "Júlio de Mesquita Filho", Brasil E-mail: suzely.moimaz@unesp.br

Tânia Adas Saliba

ORCID: https://orcid.org/0000-0003-1327-2913 Universidade Estadual Paulista "Júlio de Mesquita Filho", Brasil E-mail: tania.saliba@unesp.br

Artênio José Isper Garbin

ORCID: https://orcid.org/0000-0002-7017-8942 Universidade Estadual Paulista “Júlio de Mesquita Filho", Brasil E-mail: artenio.garbin@unesp.br

\begin{abstract}
Resumo
Os povos indígenas são alvo de preocupação global em relação a Covid-19. O objetivo neste estudo foi analisar os dados epidemiológicos da Covid-19 nos Distritos Sanitários Especiais Indígenas (DSEI) do estado de Mato Grosso e avaliar a importância da educação em saúde no combate a pandemia nas comunidades indígenas. Trata-se de um estudo observacional, ecológico, de caráter quantitativo realizado no mês de novembro de 2020 no estado de Mato Grosso. Foram coletados dados sobre a Covid-19 disponíveis nos sistemas públicos da Secretaria de Saúde Indígena (SESAI) notificados nos Distritos Sanitários Especiais Indígenas (DSEI), bem como do número de Unidades Básicas de Saúde Indígenas localizadas nos distritos do estado. Em relação ao número de Unidades Básicas de Saúde Indígena, o estado conta com 176 unidades e 51 delas apenas no Distrito de Cuiabá. A taxa de incidência do DSEI Cuiabá foi de 17.412,5 por 100.000 habitantes. Quanto a taxa de mortalidade, o DSEI Cuiabá apresentou 310,9 por 100.000 habitantes. Em relação ao Brasil, a taxa de mortalidade estava em 80,2 por 100.000 habitantes e taxa de incidência de 2934/100.000habitantes. O número de Unidades Básicas de Saúde Indígena (UBSI) em Mato Grosso está de acordo com o recomendado pelo ministério da saúde do Brasil, entretanto, as taxas de mortalidade e incidência da Covid-19 nos Distritos Sanitários Especiais Indígenas se encontram elevadas quando comparadas com as taxas de mortalidade e incidência do Brasil. Medidas de prevenção e educação em saúde são determinantes no combate á patologias respiratórias em comunidades indígenas no Brasil.
\end{abstract}

Palavras-chave: Covid-19; Indígenas; Prevenção.

\begin{abstract}
Indigenous peoples are the target of global concern in relation to Covid-19. The aim of this study was to analyze the epidemiological data of Covid-19 in the Special Indigenous Sanitary Districts (DSEI) of the state of Mato Grosso and to evaluate the importance of health education in combating the pandemic in indigenous communities. This is an observational, ecological, quantitative study carried out in November 2020 in the state of Mato Grosso. Data were collected on Covid-19 available in the public systems of the Secretariat of Indigenous Health (SESAI) notified in Special Indigenous Health Districts (DSEI), as well as the number of Basic Indigenous Health Units located in the state's districts. Regarding the number of Basic Indigenous Health Units, the state has 176 units and 51 of them only in the District of Cuiabá. The DSEI Cuiabá incidence rate was 17,412.5 per 100,000 inhabitants. Regarding the mortality rate, DSEI Cuiabá presented 310.9 per 100,000 inhabitants. In relation to Brazil, the mortality rate was 80.2 per 100,000 inhabitants and the incidence rate was $2934 / 100,000$ inhabitants. The number of Basic Indigenous Health Units (UBSI) in Mato Grosso is in line with that recommended by the Brazilian Ministry of Health, however, the mortality and incidence rates of Covid-19 in Special Indigenous Health Districts are high when compared to the
\end{abstract}


rates mortality and incidence rates in Brazil. Prevention measures and health education are crucial in combating respiratory pathologies in indigenous communities in Brazil.

Keywords: Covid-19; Indigenous; Prevention.

\section{Resumen}

Los pueblos indígenas son objeto de preocupación mundial en relación con Covid-19. El objetivo de este estudio fue analizar los datos epidemiológicos de Covid-19 en los Distritos Sanitarios Especiales Indígenas (DSEI) del estado de Mato Grosso y evaluar la importancia de la educación para la salud en el combate a la pandemia en comunidades indígenas. Se trata de un estudio observacional, ecológico y cuantitativo realizado en noviembre de 2020 en el estado de Mato Grosso. Se recolectaron datos sobre Covid-19 disponibles en los sistemas públicos de la Secretaría de Salud Indígena (SESAI) notificados en Distritos Especiales de Salud Indígena (DSEI), así como el número de Unidades Básicas de Salud Indígena ubicadas en los distritos del estado. En cuanto al número de Unidades Básicas de Salud Indígena, el estado cuenta con 176 unidades y 51 de ellas solo en el Distrito de Cuiabá. La tasa de incidencia de DSEI Cuiabá fue de $17.412,5$ por 100.000 habitantes. En cuanto a la tasa de mortalidad, DSEI Cuiabá presentó 310,9 por 100.000 habitantes. En relación con Brasil, la tasa de mortalidad fue de 80,2 por 100.000 habitantes y la tasa de incidencia fue de 2934 / 100.000 habitantes. El número de Unidades Básicas de Salud Indígena (UBSI) en Mato Grosso está en línea con lo recomendado por el Ministerio de Salud de Brasil, sin embargo, las tasas de mortalidad e incidencia de Covid-19 en Distritos Especiales de Salud Indígena son altas en comparación con las tasas. tasas de mortalidad e incidencia en Brasil. Las medidas de prevención y educación para la salud son cruciales para combatir las patologías respiratorias en las comunidades indígenas de Brasil.

Palabras clave: Covid-19; Indígena; Prevención.

\section{Introdução}

A pandemia do novo Coronavírus (Covid-19), declarada pela Organização Mundial da Saúde (OMS), em 11 de março de 2020, desencadeou uma escalada por pesquisas sobre possíveis estratégias para o seu enfrentamento. A vacina, principal meio de redução de susceptibilidade populacional e, consequentemente, a melhor forma de conter a velocidade de propagação das curvas epidêmicas, é, até o momento, inexistente para a Covid-19. (World Health Organization, 2020a; World Health Organization, 2020b; Rafaell et al., 2020). Desse modo, restam apenas as medidas de intervenção na estrutura social como forma de reduzir a velocidade de propagação da Covid-19. A necessidade de reconhecer o comportamento da doença e dos grupos de maior vulnerabilidade tomou espaço dos editoriais dos principais periódicos internacionais e nacionais. (Rafaell et al., 2020)

Os povos indígenas, e afrodescendentes historicamente, enfrentam muitas desigualdades, dentre elas, no acesso e na qualidade dos serviços recebidos. É importante lembrar que o racismo estrutural e a discriminação histórica contra essas populações, que persistem até hoje, tornam-nas mais vulneráveis aos efeitos da pandemia que a população em geral. Essas populações também têm renda mais baixa, complexas condições de vida e níveis mais baixos de escolaridade, entre outros determinantes sociais de saúde. (Organização Pan-Americana de Saúde, 2020)

Entre os povos indígenas, a evolução da doença se tornou um desafio. Mesmo que os dados oficias relatem a dinâmica de notificação, estes números podem não refletir necessariamente a extensão da pandemia. Ademais, a falta de desagregação dos dados dificulta o reconhecimento das regiões e dos povos mais afetados. Um grande problema está relacionado com os dados de indígenas que não vivem em terras indígenas homologadas, o que inclui aqueles que esperam o processo de demarcação de suas terras e também citadinos. (Instituto Socioambiental, 2020)

Sabe-se que existem diversas adversidades para o tratamento da população dos índios, como as diferenças entre os vários grupos étnicos, baixa quantidade de profissionais capacitados para o trabalho e a estrutura do local para tratamento por exemplo. (Rodrigues et al., 2018) A garantia de assistência que assegura a saúde indígena é estabelecida pelo Subsistema de Atenção à Saúde Indígena, integrado ao Sistema Único de Saúde (SUS), porém, a gestão administrativa é realizada pela Secretaria Especial de Saúde Indígena SESAI (Cardoso, 2014) Respeitando a crença e a cultura deste povo, Segundo Coimbra Júnior e Santos (2000) e Carlos Júnior (2014) a coleta e análise das informações demográficas são deficientes e necessitam de um sistema estatístico integrado aos sistemas nacionais de informação na área da saúde e que também sejam confiáveis. É de 
grande destaque da SESAI criar ações de atenção integral à saúde indígena e educação em saúde, em consonância com as políticas e os programas do SUS observando as práticas de saúde tradicionais indígena e realizar ações de saneamento e edificações de saúde indígena. (Brasil, 2020a)

Com a declaração da pandemia do novo coronavírus, a secretaria de saúde indígena criou uma página com o objetivo de divulgar a realidade da pandemia nos Distritos Sanitários Especiais indígenas. Estes números representam os dados dos 34 distritos e são validados pelo (DASI) Departamento de Atenção à Saúde Indígena. (Brasil, 2020b).

O modo de vida indígena, a organização familiar, a construção das moradias e tipo de ocupação variam de acordo com a tradição de cada povo e com o grau interação com a sociedade envolvente. De modo geral, hábitos e rituais indígenas envolvem atividades coletivas. (Cardoso et al., 2019) Os indígenas possuem menos proteção contra certos agentes etiológicos, resultando em adoecimento ou óbitos. (Brasil, 2012).

Um estudo sobre prevalência de pneumonia em crianças indígenas no Brasil encontrou associação da enfermidade com número de residentes e características físicas das casas. O número de pessoas no mesmo ambiente favorece a transmissão de patologias respiratórias. O material das moradias pode propiciar falta de circulação do ar, umidade e mofo, fatores relacionados às alergias e infecções respiratórias. (Cardoso et al., 2015) Neste sentido, pesquisas epidemiológicas voltadas aos povos indígenas são fundamentais para o monitoramento das doenças e afecções, com vistas à implementação de estratégias eficazes de promoção da saúde, controle e tratamento dos problemas existentes.

O objetivo neste estudo foi analisar os dados epidemiológicos da Covid-19 nos Distritos Sanitários Especiais Indígenas (DSEI) do estado de Mato Grosso e avaliar a importância da educação em saúde no combate a pandemia nas comunidades indígenas.

\section{Metodologia}

Trata-se de um estudo do tipo observacional, ecológico, de abordagem quantitativa que foi realizado no ano de 2020 no estado de Mato Grosso, Brasil. Nos métodos quantitativos, faz-se a coleta de dados quantitativos ou numéricos por meio do uso de medições de grandezas e obtém-se por meio da metrologia, números com suas respectivas unidades. (Pereira et al., 2018). Foram coletados dados sobre o Covid-19, disponíveis nos sistemas públicos da Secretaria de Saúde Indígena (SESAI), notificados nos Distritos Sanitários Especiais Indígenas (DSEI) do estado.

Foram analisados os dados divulgados pela Secretaria de Saúde Indígena, relacionados aos 6 distritos existentes em Mato Grosso, Brasil: Cuiabá, Vilhena, Kaiapó de Mato Grosso, Araguaia, Xingu e Xavante. As variáveis estudadas foram os números de casos suspeitos, confirmados, descartados, infectados (atual), cura clínica e óbitos. Foi pesquisado também, o número de Unidades Básicas de Saúde Indígena - UBSI encontrados em cada distrito. Foram incluídos no estudo indivíduos do sexo masculino e feminino, e de todas as faixas etárias.

Durante a pesquisa foram investigados dados secundários disponíveis em: https://saudeindigena.saude.gov.br/corona, e em páginas eletrônicas oficiais do Ministério da Saúde do Brasil. Não houve identificação de sujeitos, e, em se tratando desse tipo de pesquisa, é dispensada a apreciação em comitê de ética em pesquisa, em conformidade com a Resolução 466/2012 do Conselho Nacional de Saúde.

\section{Resultados}

O Distrito Sanitário Especial Indígena (DSEI) é a unidade gestora descentralizada do Subsistema de Atenção à Saúde Indígena (SasiSUS). No estado de Mato Grosso são localizados 6 destes distritos: Distrito Vilhena, Distrito Cuiabá, Distrito 
Xavante, Distrito Xingu, Distrito Araguaia, e Distrito Kaiapó de Mato Grosso. A Figura 1 informa as localizações destes distritos.

Figura 1. Mapa dos Distritos Sanitários Especiais Indígenas de Mato Grosso, Brasil 2020.

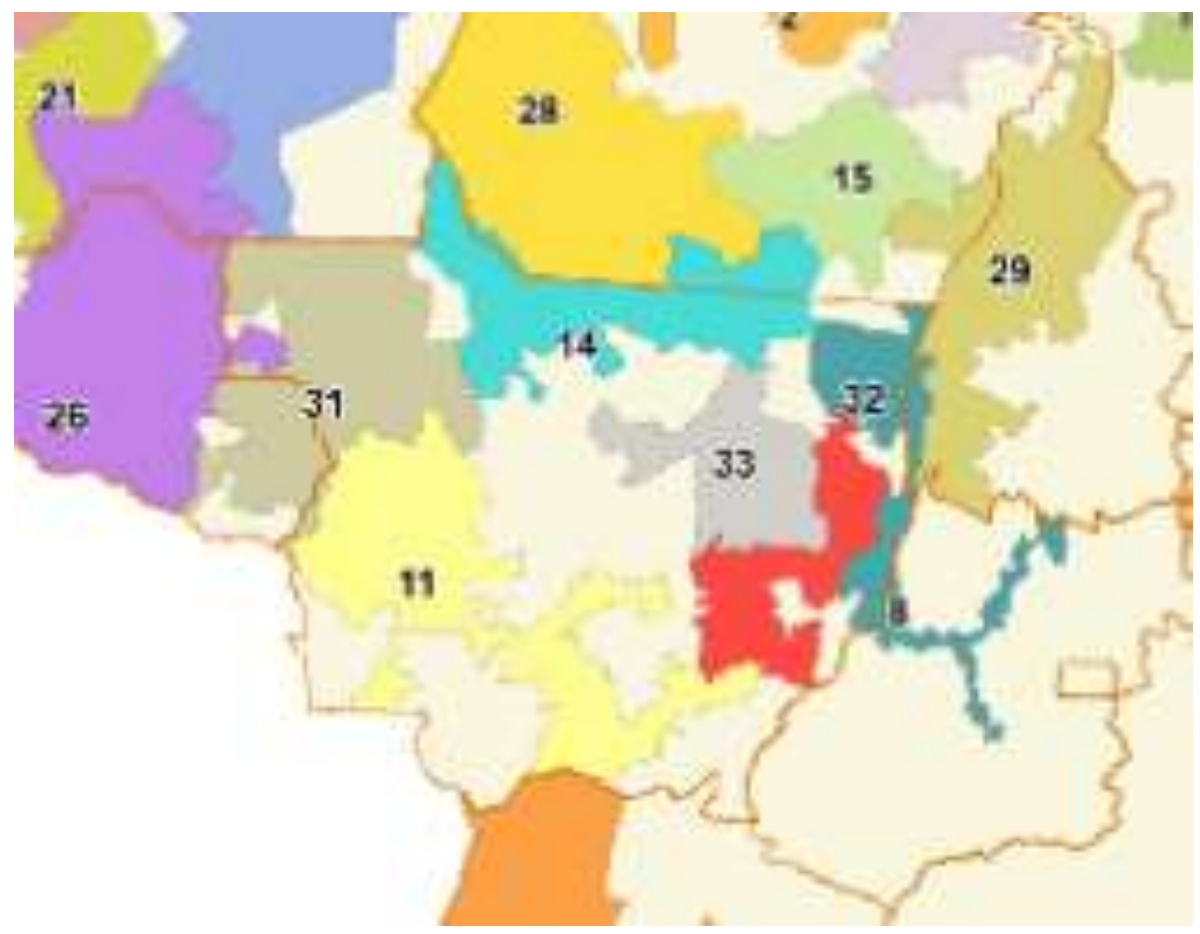

08 Distrito Araguaia

11 Distrito Cuiabá

14 Distrito Kaiapó de Mato Grosso

31 Distrito Vilhena

32 Distrito Xavante

33 Distrito Xingu

Fonte: Ministério da Saúde (2020).

É importante observar na Figura 1 que em Mato Grosso são encontrados seis Distritos Sanitários Especiais indígenas, e que estão distribuídos em cada região do estado. Estes distritos são divididos em: Araguaia, Cuiabá, Kaiapó de Mato Grosso, Vilhena, Xavante e Xingu.

De acordo com o IBGE (Instituto Brasileiro de Geografia e Pesquisa, 2020), o estado de Mato Grosso apresenta aproximadamente 3,5 milhões de habitantes. A população indígena dos DSEI é de 54399 mil. Em relação ao número de Unidades Básicas de Saúde Indígena, o estado possui 176, representados na Tabela 1. 
Tabela 1. População indígena, número de Unidades básicas de saúde Indígena dos Distritos e distribuição de habitantes por UBSI, 2020.

\begin{tabular}{|c|c|c|c|}
\hline DSEI & População & Número de UBSI & $\begin{array}{l}\text { Número } \mathrm{de} \\
\text { Habitantes por UBSI }\end{array}$ \\
\hline Vilhena & 5898 & 27 & 218 habitantes /UBSI \\
\hline Cuiabá & 7397 & 51 & 145 habitantes /UBSI \\
\hline Xavante & 22188 & 32 & 693 habitantes /UBSI \\
\hline Xingu & 8072 & 23 & 350 habitantes /UBSI \\
\hline Araguaia & 5855 & 19 & 308 habitantes /UBSI \\
\hline Kaiapó de Mato Grosso & 4989 & 24 & 207 habitantes /UBSI \\
\hline Total & 54399 & 176 & 309 habitantes /UBSI \\
\hline
\end{tabular}

Fonte: Ministério da Saúde (2020).

Na Tabela 1, é importante observar o número de habitantes e a distribuição das Unidades Básicas de Saúde Indígenas nos Distritos Sanitários Especiais Indígenas. O distrito de Xavante apresenta o maior número de habitantes, mas é o distrito de Cuiabá que concentra o maior número de Unidades Básicas de Saúde Indígena.

Os casos suspeitos, confirmados, descartados, infectados e os casos que evoluíram para cura clínica ou óbitos em todos os 6 Distritos Sanitários Especiais Indígenas (DSEI) de Mato Grosso foram notificados, e estes dados registrados até o dia 16 de novembro de 2020 estão indicados na Tabela 2.

Tabela 2. Número de casos suspeitos, confirmados, descartados, infectados, cura clínica e óbitos notificados nos DSEI, Brasil, 2020.

\begin{tabular}{l|l|l|l|l|l|l}
\hline Distrito & Suspeitos & Descartados & Confirmados & $\begin{array}{l}\text { Infectados } \\
\text { atual }\end{array}$ & $\begin{array}{l}\text { Cura } \\
\text { Clínica }\end{array}$ & Óbitos \\
\hline Vilhena & 0 & 824 & 687 & 0 & 671 & 15 \\
\hline Cuiabá & 30 & 740 & 1288 & 69 & 1188 & 23 \\
\hline Xavante & 5 & 2014 & 841 & 103 & 686 & 45 \\
\hline Xingu & 59 & 650 & 516 & 125 & 378 & 13 \\
\hline Araguaia & 11 & 353 & 266 & 0 & 259 & 5 \\
\hline Kaiapó & 12 & 414 & 843 & 0 & 839 & 4 \\
\hline Total & 121 & 5152 & 4441 & 297 & 4061 & 105 \\
\hline
\end{tabular}

Fonte: Ministério da Saúde (2020).

Na Tabela 2 é importante destacar que todos os Distritos Sanitários Especiais Indígenas registraram óbitos causados pela Covid-19. O distrito de Cuiabá registrou o maior número de casos confirmados, e o distrito de Xavante registrou o maior número de óbitos. 
A Tabela 3 apresenta a taxa de incidência e taxa de mortalidade (por 100.000 habitantes) e a letalidade por Covid-19 em indígenas assistidos pelo SASISUS, Subsistema de Atenção à Saúde Indígena por DSEI até o dia 16 de Novembro de 2020. As maiores taxas de mortalidade estão em Cuiabá, Vilhena e Xavante.

Tabela 3. Número de incidência, mortalidade e letalidade por Covid-19 nos DSEI, Brasil, 2020.

\begin{tabular}{l|l|l|l|l|l|l}
\hline DSEI & População & $\begin{array}{l}\text { Casos } \\
\text { confirmados }\end{array}$ & $\begin{array}{l}\text { Óbitos } \\
\text { confirmados }\end{array}$ & $\begin{array}{l}\text { Incidência } \\
\text { por } \\
100.000 \\
\text { habitantes }\end{array}$ & $\begin{array}{l}\text { Mortalidade } \\
\text { por 100.000 } \\
\text { habitantes }\end{array}$ & $\begin{array}{l}\text { Letalidade } \\
(\%)\end{array}$ \\
\hline Vilhena & 5.898 & 687 & 15 & 11.648 & 254,3 & 2,2 \\
\hline Araguaia & 5.855 & 266 & 5 & $4.543,1$ & 85,4 & 1,9 \\
\hline Cuiabá & 7.397 & 1.288 & 23 & $17.412,5$ & 310,9 & 1,8 \\
\hline Xavante & 22.188 & 841 & 45 & $3.790,3$ & 202,8 & 5,4 \\
\hline $\begin{array}{l}\text { Kaiapó de } \\
\text { Mato Grosso }\end{array}$ & 4.989 & 843 & 4 & $16.897,2$ & 80,2 & 0,5 \\
\hline Xingu & 8.072 & 515 & 13 & $6.380,1$ & 161,1 & 2,5 \\
\hline
\end{tabular}

Fonte: Fonte: SESAI- Ministério da Sáude.

Em geral, observa-se que a taxa de mortalidade dos DSEI do estado está alta quando comparadas com as taxas de mortalidade da população não indígena do estado de Mato Grosso e do Brasil. Em Mato Grosso, de acordo com dados do ministério da saúde, até o dia 16 de novembro de 2020, foram notificados 153.800 casos da doença em toda a população. (Indígenas e não indígenas). O número de óbitos chegou a 4025 e a taxa de mortalidade era de 115 por 100.000 habitantes e a incidência 4480 por 100.000 habitantes. Em relação ao Brasil, a taxa de mortalidade de acordo com dados do ministério da saúde até o dia 20 de Novembro de 2020 estava em 80,2 por 100.000 habitantes e taxa de incidência de 2934/100.000habitantes (Brasil, 2020c).

\section{Discussões}

O Distrito Sanitário Especial Indígena (DSEI) é a unidade gestora descentralizada do Subsistema de Atenção à Saúde Indígena (SasiSUS). Trata-se de um modelo de organização de serviços orientado para um espaço etno-cultural dinâmico, geográfico, populacional e administrativo bem delimitado que contempla um conjunto de atividades técnicas que se fundamentam em medidas racionalizadas e qualificadas de atenção à saúde. (Brasil, 2020d)

Em cada Distrito Sanitário Especial Indígena há Unidades Básicas de Saúde Indígena, que buscam atender estas populações. O número de UBSI encontradas nos Distritos Especiais Sanitários de Mato Grosso chegam a 176 unidades, sendo que 51 delas estão localizadas apenas no Distrito de Cuiabá (Brasil, 2020b). De acordo com a portaria Na2488 de 21 de Outubro de 2011 do ministério da saúde, para UBS (Unidade Básica de Saúde) é recomendado uma Unidade Básica de Saúde para até 12 mil habitantes. (Brasil, 2011). O número de Unidades Básicas de Saúde Indígena apresentou média de uma UBSI para 309 indígenas, número que está dentro do recomendado pelo Ministério da Saúde. De acordo com Ibañez e Spinelli (2002) regiões com alta concentração de leitos possibilitam a concentração de médicos, o que favorece, entre outras coisas, a oferta de serviços de saúde. 
De acordo com o boletim epidemiológico divulgado pela Secretária de Saúde Indígena, órgão do ministério da saúde responsável por cuidar da saúde indígena, todos os distritos apresentaram casos suspeitos, confirmados, descartados, infectados (atual), recuperados e óbitos causados pela Covid-19 divulgados até o dia 16 de Novembro de 2020. Os dados representam notificações de indígenas do sexo masculino e feminino e de todas as idades.

O caso suspeito é definido como o caso do indígena que saiu da aldeia e retornou apresentando quadro respiratório agudo. É definido também para aquele que teve contato com caso suspeito ou confirmado de Covid-19 e que apresentou sintomas. O caso confirmado é aquele paciente que apresentou resultado positivo nos exames laboratoriais ou por critérios clínicos. Já o Infectado atual é o caso confirmado para Covid-19, com infecção ativa, que ainda não completou 10 dias em isolamento domiciliar ou não recebeu alta médica. (Brasil, 2020a)

O caso descartado é o que apresentou resultado negativo da doença por meio de exames laboratoriais. Os recuperados, são casos confirmados que passaram por 10 dias em isolamento domiciliar, a contar da data de início dos sintomas e que estão há 24 horas assintomáticos. O número de infectados pela doença nos distritos do estado de Mato Grosso já atingiu 4441 indígenas. Em relação as mortes provocadas pela doença, ressalta-se que o número de óbitos foi de 105, representando uma taxa de $2 \%$ dos casos confirmados. (Brasil, 2020a)

O coeficiente de incidência pode demonstrar a ocorrência de novos casos em um determinado período de tempo. É possível compreender o numerador como o número de "casos novos" diagnosticados e que serão divididos pelo total de expostos. (Jekel, Elmore, \& Katz, 1999)

A taxa de Mortalidade é usada para analisar o impacto de uma doença ou condição em toda a população de uma região, sendo assim a taxa de mortalidade por covid-19 informa quantas pessoas estão morrendo por esta doença em uma determinada população. Outra medida valiosa e facilmente confundida com a taxa de mortalidade é a taxa de letalidade, que avalia o número de mortes em relação às pessoas que apresentam a doença ativa, e não em relação à população toda, ou seja, mede a porcentagem de pessoas infectadas que evoluem para óbito. (Machado et al., 2020)

De acordo com o informe epidemiológico a taxa de incidência da covid-19 na população indígena assistida pelo SASISUS, que compreende os 34 DSEI do Brasil, até 16 de novembro de 2020 foi de 4.436,2 (por 100.000 habitantes) e a taxa de mortalidade foi de 64,2 (por 100.000 habitantes). Quanto à taxa de letalidade, a região centro-oeste apresentou a maior taxa $(2,5)$, sendo 1,8 vezes maior do que taxa geral de letalidade entre os DSEI. Em Mato Grosso, As maiores taxas de incidência foram observadas nos DSEI Cuiabá (17.412,5 por 100.000 habitantes), Kaiapó do Mato Grosso (16.897,2 por 100.000 habitantes) e Vilhena (11.648,0 por 100.000 habitantes). Os DSEI que apresentaram maiores taxas de mortalidade foram Cuiabá (310,9 por 100.000 habitantes) seguido por Vilhena (254,3 por 100.000 habitantes) e Xavante (202,8 por 100.000 habitantes). Em relação a taxa de letalidade, o distrito Xavante registrou os maiores números, com uma porcentagem de 5,4\%, seguida do distrito do Xingu com 2,5\%. (Brasil, 2020a)

$\mathrm{Na}$ atenção aos povos indígenas, a mera oferta de serviços não garante a resolutividade do problema. A administração de imunizações pelo calendário vacinal da criança junto aos Terena em 2014, por exemplo, teve como objetivo cumprir a meta do Programa Nacional de Imunização. (Ribeiro, 2015) Entretanto, para mais efetividade, essa campanha poderia ter integrado um programa mais amplo de educação em saúde para a promoção integral da saúde da criança. A vacinação contra H1N1 realizada na população da etnia Guarani residente no sudeste brasileiro, por exemplo, não foi suficiente para prevenir o forte surto de síndrome gripal na região em 2016. (Cardoso et al., 2019) Pesquisas sobre surtos em aldeias indígenas durante a pandemia do H1N1 em 2009, em diferentes locais do mundo, reforçam a importância da educação em saúde feita de forma maciça pelos profissionais de saúde nas aldeias para controlar a doença. (Chidgzey et al., 2012; Pollock et al., 2009)

A vulnerabilidade dos povos indígenas, pensada em sua acepção ampliada e crítica, é potencializada quando as ações propostas pelo SUS não consideram as perspectivas desses povos na gestão do serviço, ou seja, quando as ações de saúde 
pública não são dialógicas com a população em foco (Ahmed et al., 2020). A gestão das ações em saúde para a população indígena necessita ser dialogada e deliberada com esse grupo e com as lideranças locais. (Zavaleta, 2020) Sendo assim, é importante destacar o papel do profissional da saúde, em função do diálogo e contato com estes povos. (Ribeiro \& Rossi, 2020)

\section{Conclusão}

Conclui - se que o número de Unidades básicas de Saúde Indígena (UBSI) em Mato Grosso está de acordo com o ecomendado pelo ministério da saúde do Brasil. Entretanto, as taxas de mortalidade e incidência da Covid-19 nos Distritos Sanitários Especiais Indígenas estão elevadas quando comparadas com as taxas de mortalidade e incidência do Brasil. Medidas de prevenção, promoção, educação em saúde e o diálogo entre gestores, legisladores profissionais de saúde e comunidade indígena são fatores determinantes no combate a patologias respiratórias em comunidades indígenas no Brasil. Neste trabalho, foram consultados dados secundários, o que restringe análises mais aprofundadas das taxas registradas oficialmente. Embora o estudo apresente esta limitação, ele traz à tona dados importantes para discussão sobre a saúde indígena e suas fragilidades. Novas pesquisas podem ser realizadas por meio de aplicação de questionários e inquéritos entre as populações destas comunidades.

\section{Agradecimentos}

O presente trabalho foi realizado com apoio da Coordenação de Aperfeiçoamento de Pessoal de Nível Superior Brasil (CAPES) - Código de Financiamento 001".

\section{Referências}

Ahmed, F., Ahmed, N., Pissarides, C., \& Stiglitz, J. (2020). Why inequality could spread COVID-19. The Lancet. Public Health, 5(5), e240.

Brasil. (2011). Ministério da Saúde. Portaria $n^{\circ}$ 2.488, de 21 de outubro de 2011. Aprova a Política Nacional de Atenção Básica, estabelecendo a revisão de diretrizes e normas para a organização da Atenção Básica, para a Estratégia Saúde da Família (ESF) e o Programa de Agentes Comunitários de Saúde (PACS). http://bvsms.saude.gov.br/bvs/saudelegis/gm/2011/prt2488_21_10_2011_comp.html

Brasil. (2012). Ministério da Saúde. Investigação de surto de síndrome gripal em populações indígenas, Altamira-Pará, abril-setembro de 2010. Boletim Epidemiológico da Secretaria de Vigilância em Saúde, 43(3), 11-16.

Brasil. (2020a). Ministério da Saúde. Sobre a SESAI. https://www.saude.gov.br/saude-indigena/sobre-a-sesai

Brasil. (2020b). Ministério da Saúde. Secretaria Especial de Saúde Indígena. Boletim epidemiológico da SESAI. https://saudeindigena.saude.gov.br/corona Brasil. (2020c). Ministério da Saúde. COVID-19 Painel Coronavírus. https://covid.saude.gov.br/

Brasil. (2020d). Ministério da Saúde. Secretaria Especial de Saúde Indígena. Enfrentamento da Covid-19. DSEI. https://saudeindigena.saude.gov.br/corona

Cardoso, A. M., Hortab, B. L., Santos, R. V., Escobar, A. L., Welcha, J. R., \& Coimbra Jr C. E. A. (2015). Prevalence of pneumonia and associated factors among indigenous children in Brazil: results from the First National Survey of Indigenous People's Health and Nutrition. International Health, 7, $412-419$.

Cardoso, M. D. (2014). Saúde e povos indígenas no Brasil: notas sobre alguns temas equívocos na política atual. Cadernos de Saúde Pública, 30(4), 860866.

Cardoso, A. M., Resende, P. C., Paixao, E. S., Tavares, F. G., Farias, Y. N., Barreto, C., Pantoja, L. N., Ferreira, F. L., Martins, A. L., Lima, Â. B., Fernandes, D. A., Sanches, P. M., Almeida, W., Rodrigues, L. C., \& Siqueira, M. M. (2019). Investigation of an outbreak of acute respiratory disease in an indigenous village in Brazil: contribution of Influenza A(H1N1)pdm09 and human respiratory syncytial viruses. PloS One, 14(7), e0218925.

Carlos Jr., E. A. C. (2014). Saúde e povos indígenas no Brasil: reflexões a partir do I Inquérito Nacional de Saúde e Nutrição Indígena. Cadernos de Saúde Pública, 30(4), 855-859.

Chidgzey, P. J., Davis, S., Williams, P., \& Reeve, C. (2015). An outbreak of influenza A (H1N1) virus in a remote Aboriginal community post-pandemic: implications for pandemic planning and health service policy. Australian and New Zealand Journal of Public Health, 39(1), 15-20.

Coimbra Jr., C. E. A., \& Santos, R. V. (2000). Saúde, minorias e desigualdade: algumas teias de inter-relações, com ênfase nos povos indígenas no Brasil. Ciência \& Saúde Coletiva, 5(1), 125-132. 
Research, Society and Development, v. 10, n. 1, e46510111178, 2021

(CC BY 4.0) | ISSN 2525-3409 | DOI: http://dx.doi.org/10.33448/rsd-v10i1.11178

Ibañez, N., \& Spinelli, S. P. (2002). Setor filantrópico: a experiência na operação de planos de saúde no Estado de São Paulo. In B. Negri, \& A. L. A. Viana, organizadores. O Sistema Único de Saúde em dez anos de desafio (pp. 587-606). Sobravime.

Instituto Brasileiro de Geografia e Estatística. (2020). Cidades e estados: Mato Grosso. https://www.ibge.gov.br/cidades-e-estados/mt.html?

Instituto Socioambiental. (2020). Plataforma de monitoramento da situação indígena na pandemia do novo coronavírus (Covid-19) no Brasil. https://Covid19.socioambiental.org/

Jekel, J. F., Elmore, J. G., \& Katz, D. L. (1999). Epidemiologia, bioestatística e medicina preventiva. Artes Médicas.

Machado, C. L., Ninomiya, V. Y., Shiomatsu, G. Y., \& Carvalho, R. T. (2020). Taxa de mortalidade da Covid-19: entenda esse conceito. http://coronavirus.saude.mg.gov.br/blog/81-taxa-de-mortalidade-da-covid-19

Organização Pan-Americana da Saúde. (2020). Considerações sobre povos indígenas, afrodescendentes e outros grupos étnicos durante a pandemia de COVID-19. https://iris.paho.org/bitstream/handle/10665.2/52280/OPASBRAIMSPHECOVID-19200030_por.pdf?sequence=5\&isAllowed=y

Pereira, A. S. et al. (2018). Metodologia da pesquisa científica. Ed. UAB/NTE/UFSM. https://repositorio.ufsm.br/bitstream/handle/1/15824/Lic_Computacao_ Metodologia-Pesquisa-Cientifica.pdf?sequence $=1$.

Pollock, S. L., Sagan, M., Oakley, L., Fontaine, J., \& Poffenroth, L. (2012). Investigation of a pandemic H1N1 influenza outbreak in a remote First Nations community in northern Manitoba, 2009. Canadian Journal of Public Health, 103(2), 90-93.

Rafaell, R. M. R., Neto, M., Carvalho, M. M. B., David, H. M. S. L., Acioli, S., \& Faria, M. G. A. (2020). Epidemiology, public policies and Covid-19 pandemics in Brazil: what can we expect? Revista Enfermagem UERJ, 28, e49570.

Ribeiro, A. A. (2015). O cuidado no espaço de intermedicalidade em uma aldeia indígena. Tese (Doutorado) - Universidade de São Paulo, Ribeirão Preto, SP, Brasil.

Ribeiro, A. A., \& Rossi, L. A. (2020). Pandemia covid-19 e motivações para procura pelo Sistema Único de Saúde em aldeias indígenas. Revista Brasileira de Enfermagem, 73(Suppl. 2), e20200312.

Rodrigues, F. I., Garbin, C. A. S., Moimaz, S. A. S., \& Saliba, N. A. (2018). Documentary analysis of the oral health services offered to the brazilian indigenous population. Revista Ciência Plural, 4(1), 7-21.

World Health Organization. (2020a). Naming the coronavirus disease (COVID-19) and the virus that causes it. http://who.int/emergencies/diseases/novelcoronavirus-2019/technical-guidance/naming-thecoronavirusdisease-(COVID-2019)-and-the-virusthat-causes-it

World Health Organization. (2020b). Rollings updates on coronavirus disease. https://www.who. int/emergencies/diseases/novel-coronavirus-2019/ events-asthey-happen

Zavaleta C. (2020). COVID-19: review Indigenous peoples' data. Nature, 580(7802), 185. 